\title{
Perception of flood waste management among stakeholders in Kelantan
}

\begin{abstract}
Objective: The purpose of this study is to determine the perception of stakeholder regarding the post-flood waste management system among the stakeholder authority. Methodology: This is a qualitative study and using in-depth interview. Results: The participants are officers involved in post flood from district council, SWCorp and Alam Flora. Among the criteria taken into consideration during the selection of respondents is having experience in flood waste management and involve in managing problematic areas. Conclusion: Researcher determined theme on severity of flood, waste collection, factors of failures and suggestion for improvement.
\end{abstract}

Keyword: Flood; Disaster; Waste management; Stakeholder 